\section{Explaining the aggressiveness of breast cancer in sub-Saharan African patients}

The incidence of breast cancer (BC) in sub-Saharan Africa is projected to double between 2016 and 2035. The control of the disease in the region will become an increasingly critical challenge. ${ }^{1}$ Comprehensive prevention, detection and treatment programmes are lacking; the health infrastructure is insufficient; the technical workforce is limited, and the diagnostic and treatment capacity is poor. ${ }^{2}$

Over the last 20 years, a pool of Italian cancer research centres, cancer volunteer associations and scientific societies in collaboration with the Tanzanian political and health authorities have created a pathology laboratory (that they considered to be a core element of a cancer care and control programme) and a medical oncology unit at the Bugando Medical Centre (BMC) of Mwanza, the largest referral hospital in the Lake zone of north-western Tanzania. This endeavour is known as the Mwanza Cancer Project. ${ }^{3}$

Based on the idea that offering scientific tutoring and access to research programmes can increase the capacity building of local healthcare professionals, these have been involved in several research projects under way in Italy. One of these has been designed to perform a comparative biological characterisation of BC from a series of Tanzanian patients and a series of Italian patients. ${ }^{4}$ For the former, the pathological processing and reporting of surgical specimens have been performed at the pathology laboratory of the BMC, while oestrogen receptor (ER), progesterone receptor (PR) and androgen receptor (AR) status, human epidermal growth factor receptor 2 expression and proliferative activity (Ki67) have been assessed at the Bioscience Laboratory of the Istituto Scientifico Romagnolo per lo Studio e la Cura dei Tumori, the Italian cancer research centre where the Mwanza Cancer Project was developed.

According to the results of the study, the histopathological and molecular characteristics of BC were substantially different between Tanzanian and Italian patients. The former presented more often with advanced (stages II-IV, 89\% vs $49 \%$ ), poorly differentiated (grade III, $67 \%$ vs $46 \%$ ), ER-negative (52\% vs $20 \%)$, triple-negative (28\% vs $12 \%$ ) and highly proliferating tumours (high Ki-67, $77 \%$ vs $43 \%$ ). The findings from Tanzanian patients were roughly consistent with those of other previous studies.

The greater prevalence of ER-negative and triple-negative $\mathrm{BC}$ would be a serious problem both for the prognostic implications and for the fact that sub-Saharan African patients with BC are usually treated with endocrine therapy (if available and accessible) without knowledge of the hormone receptor status, ${ }^{4}$ which means overtreatment of many patients combined with waste of resources in a low-resource setting.

The delay in diagnosis may well be responsible not only for the more advanced disease stage but also for the lower degree of differentiation, because the biological characteristics of $\mathrm{BC}$ worsen progressively during the preclinical phase. This is the case, for example, for the regional metastatic potential and the histological grade.

The aggressive histological features of BC in sub-Saharan African patients may also be related to genetic variation. We believe that the potential influence of selected nucleotide polymorphisms in genes on the biological aggressiveness of BC in sub-Saharan African patients will be the subject of increasing attention, in parallel with studies on the relationship between functional genetic variants and the inter-racial variability in pharmacokinetics of BC chemotherapy drugs.

However, the results of some African studies are compatible with the hypothesis that a third factor might be responsible-at least in part-of the molecular characteristics of BC in sub-Saharan Africa, that is, the biases deriving from preanalytical problems with tissue collection and processing, in particular from problems of underfixation or overfixation of surgical specimens. ${ }^{5-8}$ These studies, in which standardised methods for collection, processing and reporting of specimens have been prospectively used, have reported that the frequency of Kenyan, Ethiopian and South African patients with ER-negative $\mathrm{BC}$ and triple-negative $\mathrm{BC}$ may be comparable with that found among patients from the Western countries. The authors have defined the standardisation of the preanalytic process and the implementation of quality assurance schemes as urgent needs. ${ }^{67}$ They have also drawn attention to the fact that most African studies reporting a disproportionately high prevalence of patients with ER-negative $\mathrm{BC}$ had a retrospective design. ${ }^{6}$

To add further evidence to the existing African literature on the relationship between the level of standardisation of the

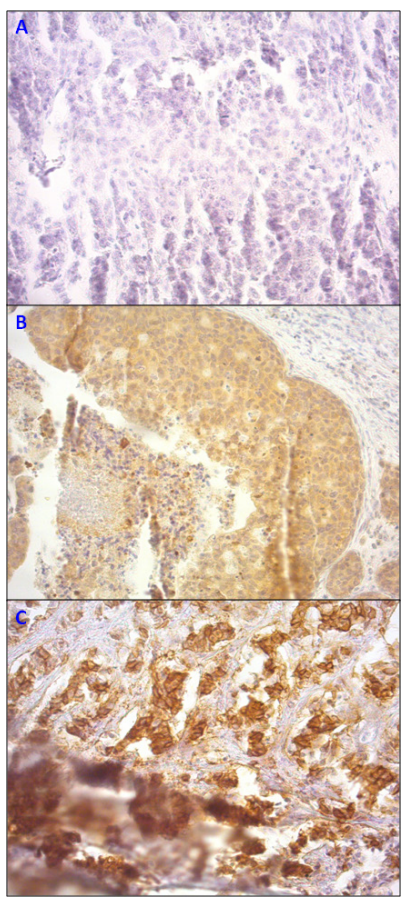

Figure 1 Invasive breast cancer tissues from a Tanzanian patient with non-specific and inhomogeneous expression of hormone receptors, $(A)$ oestrogen receptor; (B) progesterone receptor and $(C)$ human epidermal growth factor receptor 2 (20x magnification), due to preanalytical problems and high section thickness. The staining is diffuse, and not specific, and there are artefacts in all the samples.

preanalytic process and the distribution by BC subtype, two of us (SB and MP) have reviewed the samples of the original immunohistochemical preparations from the Italian-Tanzanian study. ${ }^{4}$ They have found that about one-third of specimens from Tanzania were not evaluable and showed staining artefacts reflecting limitations in fixation and paraffin-embedding procedures. The figure 1 shows the specimens of a patient that have been reclassified as not evaluable because of inhomogeneous staining for ER, PR and ERB2.

Unless one suggests that immunohistochemical studies of BC in sub-Saharan African patients should be performed on women who have migrated to the Western world, the construction of a tutoring system and the promotion of quality assurance are the only resolutions. In developed countries, health authorities have established criteria for the minimum infrastructure that enable pathology laboratories to maintain minimum quality requirements. In sub-Saharan Africa, this approach remains valid but needs to be adapted to the local environment. Since workforce, infrastructure and technologies are 
underdeveloped and their upgrade is difficult, tutoring and quality assurance are more important than elsewhere. Scientific organisations and cancer centres of the developed world could make an impact by implementing this strategy, especially in conjunction with the conduct of international collaborative studies.

These actions may complement local initiatives like, for example, the multidisciplinary workshop held in Nairobi in 2012 as part of a multicentre study on the prevalence of triple-negative $\mathrm{BC},{ }^{6}$ where the preanalytical factors impacting on the immunohistochemical process and the possible solutions were identified, and training in core biopsy was provided.

These would be the major steps forward in the characterisation of BC (and other malignancies) in sub-Saharan Africa. Before concluding that BC in sub-Saharan African patients has unfavourable biological characteristics compared with Caucasian patients, and before dealing with the serious implications of this, further studies with a standardised preanalytical phase are needed.

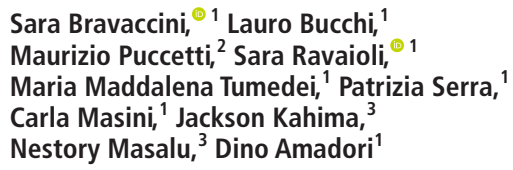

${ }^{1}$ Istituto Scientifico Romagnolo per lo Studio e la Cura dei Tumori (IRST) IRCCS, Meldola, Italy

${ }^{2}$ Azienda Unità Sanitaria Locale (AUSL) Imola, Imola, Italy

${ }^{3}$ Bugando Medical Center, Mwanza, United Republic of Tanzania

Correspondence to Dr Lauro Bucchi, Istituto Scientifico Romagnolo per lo Studio e la Cura dei Tumori (IRST) IRCCS, Meldola 47014, Italy; lauro. bucchi@irst.emr.it

Handling editor Dhirendra Govender.

Contributors SB and DA conceived the study. SB and LB wrote the manuscript. PS and CM collected the data. JK and NM enrolled the Tanzanian patients with breast cancer. MMT and SR performed the immunohistochemical analyses. SB and MP read the slides of immunohistochemical preparations. All authors reviewed the final manuscript.

Competing interests None declared.

Patient consent for publication Not required.

Ethics approval The study was approved by the ethics committee at the IRST (ID: IRST100.37).

Provenance and peer review Not commissioned; externally peer reviewed.

(C) Author(s) (or their employer(s)) 2019. No commercial re-use. See rights and permissions. Published by BMJ.

\section{Check for updates}

To cite Bravaccini S, Bucchi L, Puccetti M, et al. J Clin Pathol 2019;72:723-724.

Received 3 July 2019

Revised 5 August 2019

Accepted 6 August 2019
Published Online First 13 August 2019

J Clin Pathol 2019;72:723-724.

doi:10.1136/jclinpath-2019-206071

\section{REFERENCES}

1 International Agency for Research on Cancer, World Health Organisation. Breast cancer awareness month. breast cancer survival in sub-Saharan Africa. Available: https://www.iarc.fr/wp-content/uploads/2018/07/ABCDO_QA_Oct2016.pdf [Accessed 2 Jul 2019].

2 Morhason-Bello I0, Odedina F, Rebbeck TR, et al. Challenges and opportunities in cancer control in Africa: a perspective from the African Organisation for Research and Training in Cancer. Lancet Oncol 2013; 14:e142-51.

3 Amadori D, Serra P, Bucchi L, et al. The Mwanza cancer project. Lancet Oncol 2016;17:146-8.

4 Amadori D, Serra P, Bravaccini S, et al. Differences in biological features of breast cancer between Caucasian (Italian) and African (Tanzanian) populations. Breast Cancer Res Treat 2014;145:177-83.

5 McCormack VA, Joffe $M$, van den Berg $E$, et al. Breast cancer receptor status and stage at diagnosis in over 1,200 consecutive public hospital patients in Soweto, South Africa: a case series. Breast Cancer Res 2013;15.

6 Sayed S, Moloo Z, Bird P, et al. Breast cancer diagnosis in a resource poor environment through a collaborative multidisciplinary approach: the Kenyan experience. J Clin Pathol 2013;66:307-11.

7 Sayed S, Moloo Z, Wasike R, et al. Is breast cancer from sub Saharan Africa truly receptor poor? Prevalence of ER/PR/HER2 in breast cancer from Kenya. Breast 2014;23:591-6.

8 Hadgu E, Seifu D, Tigneh W, et al. Breast cancer in Ethiopia: evidence for geographic difference in the distribution of molecular subtypes in Africa. BMC Womens Health 2018;18:40 